\title{
Low Voltage STEM Observation in the Latest FE-SEM
}

Naoki Sakamoto, Shuichi Takeuchi, Kazuhiro Koyama, Masahiro Sasajima, Hirofumi Sato

Hitachi High-Technologies Corp., 882, Ichige, Hitachinaka, Ibaraki 312-8504, Japan

Ultra high resolution FE-SEM has been broadly utilized for semiconductors and nano materials as an advanced analytical tool. High efficiency signal detection capability and high resolution, high contrast imaging are most effective for research on compositional materials in the field of material science and engineering.

In this study, newly developed STEM holder for FE-SEM low accelerating voltage observation having capabilities of both Bright Field STEM (BF-STEM) and Dark Field STEM (DF-STEM) is used. It is well known that low voltage STEM observation on FE-SEM provides high contrast imaging of low Z compositional materials compared with that of STEM observation on TEM. The newly developed specimen holder with DF-STEM detection system can also be simultaneously used when BF-STEM signal is detected[1,2].

Figure 1 shows the schematic of the BF/DF-STEM detection system for FE-SEM. The detection angle of BF signal is controlled by the STEM aperture and inner crystal information on specimen would be obtained through BF signals generally, while newly developed DF-STEM detection system would have the movable detection element which can be vertically traversed to change the detection angle of DFSTEM signals, so that we can clearly obtain an optimal Z-contrast image.

Figure 2 shows the BF-STEM (a) and DF-STEM (b) imaging of carbon nano tube (CNT) containing iron particles. Both images are of the same field of view at accelerating voltage of $30 \mathrm{kV}$. In the BFSTEM Image (a), not only iron and CNT contrasts but also diffraction contrast reflecting the crystal structure of CNT wall can be clearly observed. In the DF-STEM Image (b), high Z-contrast image is clearly observed by using of the movable detection element which enables us to obtain the optimized position. On top of that, highly precision EDS analysis utilizing the Z-contrast image on the DF-STEM is also available.

[1] S Watanabe et al, J. Electron Microsc. 59(Suppl1) (2003) p.196

[2] S Takeuchi et al, IMC16, Sapporo Conference Proceedings (2006) p.660.CD. 


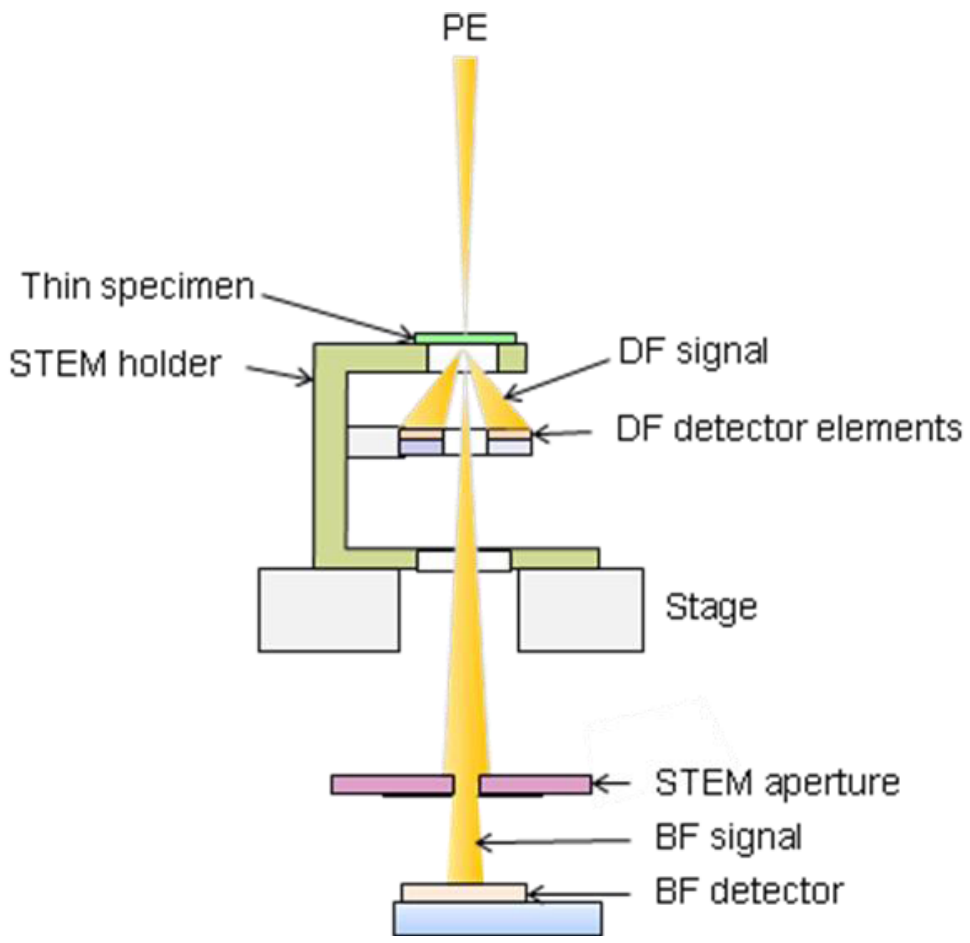

Figure 1. BF/DF-STEM detection system
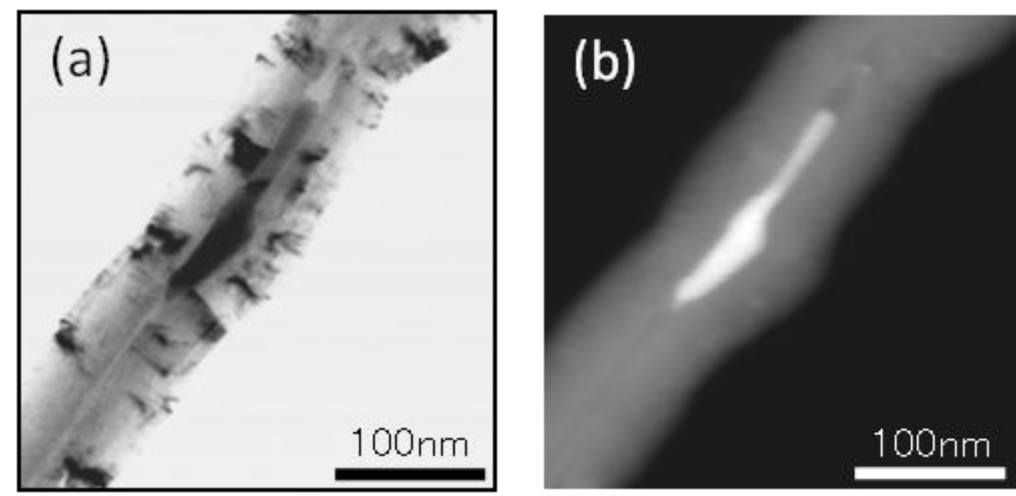

Figure 2. Low voltage STEM observation of CNT (a) BF-STEM (b) DF-STEM 